\title{
REMARKS ON THE CONSISTENCY OF UPWIND SOURCE AT INTERFACE SCHEMES ON NONUNIFORM GRIDS
}

\section{Chiara Simeoni}

work in progress with G. Puppo and M. Semplice

\author{
Laboratoire de Mathématiques J.A. Dieudonné \\ Université Nice Sophia Antipolis, France \\ e-mail : simeoni@unice.fr \\ http://math.unice.fr/laboratoire/fiche\&id $=257$
}

\section{preliminary study of the supra-convergence of finite volume schemes for conservation laws with geometrical source terms}

- upwind interfacial discretizations for nonuniform time/space grids

- inconsistent characteristics of the (local) truncation error

- notion of (global) consistency, related to the well-balance property

- convergence theory at optimal rates according to Wendroff \&White

- computational performance as adaptive meshing yields an extra stabilization against the nonlinear response over shock regions 
formal analysis for scalar (linear) advection/transport balance equations

$$
\begin{aligned}
& \partial_{t} u+\partial_{x} A(u)+b(u) z^{\prime}(x)=0, \quad t \in \mathbb{R}^{+}, x \in \mathbb{R} \\
& u(0, x)=u_{0}(x) \in L^{p}(\mathbb{R}) \cap L^{\infty}(\mathbb{R}), \quad 1 \leq p<+\infty
\end{aligned}
$$

where $a(u)=A^{\prime}(u)=a>0, z^{\prime} \in L^{p}(\mathbb{R}) \cap L^{\infty}(\mathbb{R})$ and $b \in C^{1}(\mathbb{R})$

and the stationary solutions are described by

$$
D(u(x))+z(x)=C^{s t}, \quad D^{\prime}(u)=\frac{a(u)}{b(u)} \in L^{\infty}(\mathbb{R})
$$

(with $D$ strictly monotonic for the existence of a unique Lipschitz continuous steady state)

difficulties and limits : restriction to geometrical source terms for the extended notation as (non conservative) fluxes; physical applications with negligible fluxes (groundwater models, nonlinear age-dependent population dynamics, stochastic processes with multiplicative noise); improvements by mesh adaptivity may reduce because modified schemes loose critical features (conservation form, well-balancing, ...) 
numerical issues : accurate computation of non-constant steady states, occurring for the balance between source term and internal forces; unstructured grids required for multi-dimensional problems incorporating composite physical geometries

- B.D. Rogers, A.G.L. Borthwick, P.H. Taylor, Mathematical balancing of flux gradient and source terms prior to using Roe's approximate Riemann solver, J. Comput. Phys. (2003)

- M.J. Castro, P. Garcia-Navarro, The application of a conservative grid adaptation technique to $1 \mathrm{D}$ shallow water equations, Math. Comput. Modelling (2001)

- S. Karni, A. Kurganov, G. Petrova, A smoothness indicator for adaptive algorithms for hyperbolic systems, J. Comput. Phys. (2002)

analytical studies : recent theoretical advances on adaptive techniques for mesh refinement; consistency properties of finite volume schemes setting on nonuniform grids with respect to (strong) convergence

- G. Puppo, M. Semplice, Numerical entropy and adaptivity for finite volume schemes, Commun. Comput. Phys. (2011)

- C. Arvanitis, A.I. Delis, Behavior of finite volume schemes for hyperbolic conservation laws on adaptive redistributed spatial grids, J. Sci. Comput. (2006)

- C. Arvanitis, Ch. Makridakis, N.I. Sfakianakis, Entropy conservative schemes and adaptive mesh selection for hyperbolic conservation laws, J. Hyperbolic Differ. Equ. (2010) 
numerical simulations for smooth data with periodic boundary conditions

experimental errors at time $T=1.5$, with $a=0.5, b=1.0$ and $C F L=0.9$ the nonuniform meshes for the computation are arbitrarily generated (not compatible with some $L^{p}$-type regularity condition)

\begin{tabular}{|c|c|c|c|c|c|c|}
\hline cells & \multicolumn{2}{|c|}{$\|e(t)\|_{L^{1}}$} & \multicolumn{2}{c|}{$\|e(t)\|_{L^{2}}$} & \multicolumn{2}{c|}{$\|e(t)\|_{L^{\infty}}$} \\
\hline & & rates & & rates & & rates \\
\hline 30 & $0.313172 \mathrm{E}-01$ & & $0.365947 \mathrm{E}-01$ & & $0.596413 \mathrm{E}-01$ & \\
\hline 60 & $0.147558 \mathrm{E}-01$ & 1.105 & $0.174315 \mathrm{E}-01$ & 1.109 & $0.251566 \mathrm{E}-01$ & 1.173 \\
\hline 120 & $0.724135 \mathrm{E}-02$ & 1.071 & $0.777632 \mathrm{E}-02$ & 1.073 & $0.138610 \mathrm{E}-01$ & 1.132 \\
\hline 240 & $0.350149 \mathrm{E}-02$ & 1.055 & $0.374801 \mathrm{E}-02$ & 1.056 & $0.556219 \mathrm{E}-02$ & 1.111 \\
\hline 480 & $0.181983 \mathrm{E}-02$ & 1.043 & $0.198378 \mathrm{E}-02$ & 1.042 & $0.264091 \mathrm{E}-02$ & 1.108 \\
\hline 960 & $0.732448 \mathrm{E}-03$ & 1.023 & $0.781343 \mathrm{E}-03$ & 1.029 & $0.824566 \mathrm{E}-03$ & 1.089 \\
\hline
\end{tabular}

Table: standard (first order) scheme on highly nonuniform grids

- nonuniform mesh with strong inhomogeneity of the cells' size :

$$
h>0, \gamma \gg 1, \quad \Delta x_{i}=\left\{\begin{array}{cc}
h & i=2 k \\
\gamma h & i=2 k+1
\end{array}, k \in \mathbb{Z}\right.
$$


accuracy test for the Saint-Venant equations of shallow waters through the kinetic scheme with reflections

- B. Perthame, C. Simeoni, A kinetic scheme for the Saint-Venant system with a source term, Calcolo (2001)
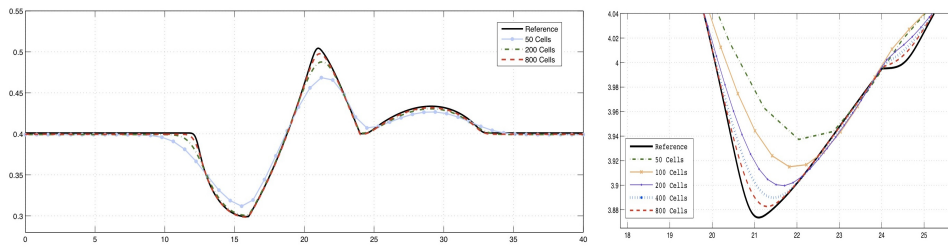

Figure: continuous solutions with discontinuity in the derivatives

\begin{tabular}{|c|c|c|c|c|c|c|}
\hline cells & \multicolumn{2}{|c|}{$\|e(t)\|_{L^{1}}$} & \multicolumn{2}{c|}{$\|e(t)\|_{L^{2}}$} & \multicolumn{2}{c|}{$\|e(t)\|_{L^{\infty}}$} \\
\hline & & rates & & rates & & rates \\
\hline 50 & 0.2251 & & 0.0768 & & 0.0460 & \\
\hline 100 & 0.1870 & 0.2673 & 0.0637 & 0.2682 & 0.0466 & -0.0198 \\
\hline 200 & 0.0927 & 1.0126 & 0.0309 & 1.0456 & 0.0224 & 1.0590 \\
\hline 400 & 0.0492 & 0.9134 & 0.0163 & 0.9218 & 0.0113 & 0.9849 \\
\hline 800 & 0.0259 & 0.9254 & 0.00874 & 0.8976 & 0.00570 & 0.9863 \\
\hline 1600 & 0.0157 & 0.7197 & 0.00627 & 0.4802 & 0.00388 & 0.558 \\
\hline
\end{tabular}

Table: experimental errors at time $T=1.0$ for parabolic source term 
finite volume schemes because of the conservation property, possible discrete versions of the entropy inequalities, and implementation with low regularity external fields (integral formulation)

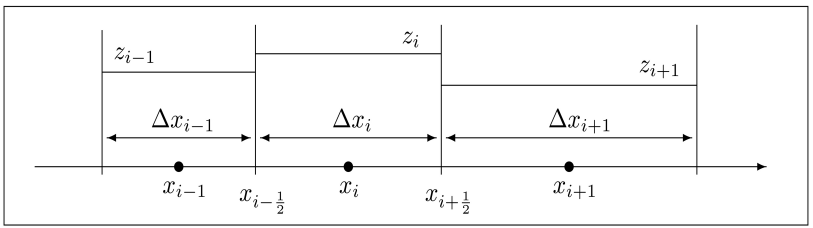

FIGURE 1. piecewise constant reconstruction on the spatial mesh

non-degeneracy constraint for nonuniform grids :

$$
\exists \alpha, \beta>0 / \alpha \Delta x_{i+1} \leq \Delta x_{i} \leq \beta \Delta x_{i+1}, \forall i \in \mathbb{Z}
$$

variable time-step $\Delta t_{n}=t_{n+1}-t_{n}, n \in \mathbb{N}$

cell-centered discrete unknowns

$$
v_{i}^{n} \approx \frac{1}{\Delta x_{i}} \int_{C_{i}} u\left(t_{n}, x\right) d x=u\left(t_{n}, x_{i}\right)+\mathcal{O}\left(h^{2}\right)
$$

characteristic parameters $h=\max _{i \in \mathbb{Z}} \Delta x_{i}$ and $\Delta t \overline{\bar{a}} \sup _{n \in \mathbb{N}} \Delta t_{n}$ 
the simplest explicit (three points) interfacial upwind (and eventually well-balanced) scheme reads

$$
\frac{v_{i}^{n+1}-v_{i}^{n}}{\Delta t_{n}}+a \frac{v_{i}^{n}-v_{i-1}^{n}}{\Delta x_{i}}+b\left(v_{i}^{n}\right) \frac{z_{i}-z_{i-1}}{\Delta x_{i}}=0
$$

together with initial data and boundary conditions

- the standard CFL-condition on the (local) ratio $\Delta t_{n} / \Delta x_{i}$ guarantees the numerical stability (adjusted to include the numerical source term)

- S. Osher, R. Sanders, Numerical approximations to nonlinear conservation laws with locally varying time and space grids, Math. Comp. (1983)

- extension to multi-dimensional finite volumes through the splitting of one-dimensional schemes (with application to linear systems)

- M. Ben-Artzi, J. Falcovitz, An upwind second-order scheme for compressible duct flows, SIAM J. Sci. Statist. Comput. (1986)

- P.L. Roe, Upwind differencing schemes for hyperbolic conservation laws with source terms, Lecture Notes in Math. (1987)

- A. Bermudez, M.E. Vazquez, Upwind methods for hyperbolic conservation laws with source terms, Computers \& Fluids (1994) 
for the cell-averages $g_{i}=\frac{1}{\Delta x_{i}} \int_{C_{i}} g(x) d x$ of any function $g \in W^{2, p}$, $1 \leq p<+\infty$, thanks to the symmetry of cell-centered integrals

$$
\begin{aligned}
g_{i} & =g\left(x_{i}\right)+\frac{1}{\Delta x_{i}} \int_{C_{i}} g^{\prime \prime}(\xi(x)) \frac{\left(x-x_{i}\right)^{2}}{2} d x \\
& =g\left(x_{i-\frac{1}{2}}\right)+g^{\prime}\left(x_{i-\frac{1}{2}}\right) \frac{\Delta x_{i}}{2}+\mathcal{O}\left(h,\left\|g^{\prime \prime}\right\|_{L^{p}}\right) \\
g_{i}-g_{i-1} & =g^{\prime}\left(x_{i}\right)\left(\frac{\Delta x_{i-1}}{2}+\frac{\Delta x_{i}}{2}\right)+\frac{1}{\Delta x_{i}} \int_{C_{i}} g^{\prime \prime}(\eta(x)) \Theta(x) d x
\end{aligned}
$$

with $\Theta(x)=\left(\xi(x)-x_{i}\right)\left(\left(x-x_{i-\frac{3}{2}}\right)-\frac{\Delta x_{i-1}}{\Delta x_{i}}\left(x-x_{i-\frac{1}{2}}\right)\right)$

for some $\xi(x), \eta(x) \in C_{i} \cup C_{i-1}$

providing the correct approximation on nonuniform meshes

- formal estimations about the consistency error

- additional condition $b(u)>0$ for the stationary equations

- smooth solutions for which expansions can be performed 
the (local) truncation error is evaluated by returning the analytical solution into the discrete formulation, with $u_{i}^{n}=u\left(t_{n}, x_{i}\right)$

$$
T_{i}^{n}=\partial_{t} u_{i}^{n}+\frac{\Delta x_{i-1}+\Delta x_{i}}{2 \Delta x_{i}}\left[a \partial_{x} u_{i}^{n}+b\left(u_{i}^{n}\right) z^{\prime}\left(x_{i}\right)\right]+\mathcal{O}(\Delta t, h)
$$

revealing a lack of consistency with the underlying balance equation

the space-step $\Delta x_{i}$ could be very different from the length of an interfacial interval $\left|x_{i}-x_{i-1}\right|=\frac{\Delta x_{i-1}}{2}+\frac{\Delta x_{i}}{2}$, and the pointwise consistency error does not vanish : unless the spatial mesh is quasi-uniform, namely $\Delta x_{i-1}=\Delta x_{i}+\mathcal{O}\left(h^{2}\right)$, it seems that convergence by the Lax theorem cannot be expected, or at least a significant reduction in the rates occurs...

- J.D. Hoffman, Relationship between the truncation errors of centered finite-difference approximations on uniform and nonuniform meshes, J. Comput. Phys. (1982)

- E. Turkel, Accuracy of schemes with nonuniform meshes for compressible fluid flows, Appl. Numer. Math. (1986)

- J. Pike, Grid adaptive algorithms for the solution of the Euler equations on irregular grids, J. Comput. Phys. (1987) 
formal accuracy of finite volume schemes is actually maintained on nonuniform meshes, because of the supra-convergence phenomenon

- investigated first for homogeneous hyperbolic conservation laws

- A.N. Tikhonov, A.A. Samarsky, On the theory of homogeneous difference schemes, Outlines Joint Sympos. Partial Differential Equations (Novosibirsk, 1963)

- H.-O. Kreiss, T.A. Manteuffel, B. Swartz, B. Wendroff, A.B. White Jr., Supra-convergent schemes on irregular grids, Math. Comp. (1986)

- B. Wendroff, A.B. White Jr., A supraconvergent scheme for nonlinear hyperbolic systems, Comput. Math. Appl. (1989)

??? (fully discrete) convergence at optimal rates for smooth solutions of upwind schemes for linear equations on two-dimensional triangulations

- B. Després, Lax theorem and finite volume schemes, Math. Comp. (2004)

- B. Després, An explicit a priori estimate for a finite volume approximation of linear advection on non-Cartesian grids, SIAM J. Numer. Anal. (2004)

??? comprehensive interpretation of a priori error estimates, based on the Kuznetsov's theory, for (scalar) nonlinear problems with low regularity

- B. Cockburn, P.-A. Gremaud, A priori error estimates for numerical methods for scalar conservation laws. II. Flux-splitting monotone schemes on irregular Cartesian grids,

Math. Comp. (1997) 
the discrete unknowns (and external fields) are replaced by

$$
v_{i}^{n} \approx \frac{1}{\Delta x_{i}} \int_{C_{i}}\left[u\left(t_{n}, x\right)+\frac{\Delta x_{i}}{2} \partial_{x} u\left(t_{n}, x\right)\right] d x, \quad n \in \mathbb{N}, i \in \mathbb{Z}
$$

with a first order correction to compensate the truncation error, since it represents precisely the discrepancy between cell-centered and interfacial averages, which do not coincide for nonuniform grids

- D. Bouche, J.-M. Ghidaglia, F. Pascal, Error estimate and the geometric corrector for the upwind finite volume method applied to the linear advection equation, SIAM J. Numer. Anal. (2005)

the modified equation of the scheme for the new reconstruction reads

$$
\begin{aligned}
R_{i}^{n}= & \partial_{t} u_{i}^{n}+a \partial_{x} u_{i}^{n}+b\left(u_{i}^{n}\right) z^{\prime}\left(x_{i}\right)+ \\
& +\frac{\Delta x_{i-1}}{2}\left[a \partial_{x x} u_{i}^{n}+b\left(u_{i}^{n}\right) z^{\prime \prime}\left(x_{i}\right)\right] \frac{\Delta x_{i-1}+\Delta x_{i}}{2 \Delta x_{i}}+ \\
& +\frac{\Delta x_{i}}{2}\left[\partial_{t x} u_{i}^{n}+b^{\prime}\left(u_{i}^{n}\right) z^{\prime}\left(x_{i}\right) \partial_{x} u_{i}^{n}\right]+\mathcal{O}(\Delta t, h)
\end{aligned}
$$

for $u_{i}^{n}=u\left(t_{n}, x_{i}\right)$, with extra terms involving (admissible) higher regularity of the solution, and bounded through the mesh's condition 
first order approximation justifies to use

$$
E_{i}^{n}=v_{i}^{n}-u\left(t_{n}, x_{i}\right)-\frac{\Delta x_{i}}{2} \partial_{x} u\left(t_{n}, x_{i}\right), \quad S_{i}^{n}=z_{i}-z\left(x_{i}\right)-\frac{\Delta x_{i}}{2} z^{\prime}\left(x_{i}\right)
$$

as reference quantities for error analysis : fixing $b(u)=b>0$ to avoid inessential technicality, the typical stability equation of the scheme

$$
E_{i}^{n+1}=E_{i}^{n}-a \frac{\Delta t_{n}}{\Delta x_{i}}\left(E_{i}^{n}-E_{i-1}^{n}\right)-b \frac{\Delta t_{n}}{\Delta x_{i}}\left(S_{i}^{n}-S_{i-1}^{n}\right)-\Delta t_{n} R_{i}^{n}
$$

provides convergence with optimal rates for (regular) nonuniform grids

- diffusive and dispersive characteristics of the numerical method

- the truncation error always vanishes for the simulation of steady states, for the well-balance property (besides an overall stability)

- conservative schemes perform substantially better on unstructured meshes in comparison to those not preserving some special structures even for uniform meshes

- O.V. Vasilyev, High order finite difference schemes on non-uniform meshes with good conservation properties, J. Comput. Phys. (2000) 
for many applications to real systems, the advection may become negligible, so that the external fields dominate over the fluxes...

\begin{tabular}{|c|c|c|c|c|c|c|}
\hline cells & \multicolumn{2}{|c|}{$\|e(t)\|_{L^{1}}$} & \multicolumn{2}{c|}{$\|e(t)\|_{L^{2}}$} & \multicolumn{2}{c|}{$\|e(t)\|_{L^{\infty}}$} \\
\hline & & rates & & rates & & rates \\
\hline 30 & $0.208333 \mathrm{E}+01$ & & $0.143466 \mathrm{E}+01$ & & $0.151412 \mathrm{E}+01$ & \\
\hline 60 & $0.229167 \mathrm{E}+01$ & -0.138 & $0.152438 \mathrm{E}+01$ & -0.088 & $0.275635 \mathrm{E}+01$ & -0.860 \\
\hline 120 & $0.260417 \mathrm{E}+01$ & -0.161 & $0.151382 \mathrm{E}+01$ & -0.039 & $0.253439 \mathrm{E}+01$ & -0.370 \\
\hline 240 & $0.255208 \mathrm{E}+01$ & -0.098 & $0.155620 \mathrm{E}+01$ & -0.039 & $0.144264 \mathrm{E}+01$ & 0.023 \\
\hline 480 & $0.252604 \mathrm{E}+01$ & -0.069 & $0.157700 \mathrm{E}+01$ & -0.034 & $0.202358 \mathrm{E}+01$ & -0.105 \\
\hline 960 & $0.248698 \mathrm{E}+01$ & -0.051 & $0.159362 \mathrm{E}+01$ & -0.030 & $0.176882 \mathrm{E}+01$ & -0.045 \\
\hline
\end{tabular}

Table: standard (first order) scheme on highly nonuniform grid for $a=0.005$

\begin{tabular}{|c|c|c|c|c|c|c|}
\hline cells & \multicolumn{2}{|c|}{$\|e(t)\|_{L^{1}}$} & \multicolumn{2}{c|}{$\|e(t)\|_{L^{2}}$} & \multicolumn{2}{c|}{$\|e(t)\|_{L^{\infty}}$} \\
\hline & & rates & & rates & & rates \\
\hline 30 & $0.308642 \mathrm{E}-01$ & & $0.156844 \mathrm{E}+00$ & & $0.254948 \mathrm{E}-01$ & \\
\hline 60 & $0.169753 \mathrm{E}-01$ & 0.862 & $0.887122 \mathrm{E}-01$ & 0.818 & $0.164315 \mathrm{E}-01$ & 1.108 \\
\hline 120 & $0.925926 \mathrm{E}-02$ & 0.868 & $0.424803 \mathrm{E}-01$ & 0.939 & $0.787522 \mathrm{E}-02$ & 1.093 \\
\hline 240 & $0.462963 \mathrm{E}-02$ & 0.912 & $0.216954 \mathrm{E}-01$ & 0.953 & $0.384303 \mathrm{E}-02$ & 1.075 \\
\hline 480 & $0.231481 \mathrm{E}-02$ & 0.934 & $0.127825 \mathrm{E}-01$ & 0.905 & $0.189368 \mathrm{E}-02$ & 1.062 \\
\hline 960 & $0.115138 \mathrm{E}-02$ & 0.949 & $0.739794 \mathrm{E}-02$ & 0.882 & $0.698813 \mathrm{E}-02$ & 1.055 \\
\hline
\end{tabular}

Table: modified (first order) scheme on highly nonuniform grid for $a=0.005$

- Th. Katsaounis, C. Simeoni,Three-points interfacial quadrature for geometrical source terms on nonuniform grids, Calcolo (2011) 
steady states for sinusoidal source term : adaptive techniques customarily generate quasi-uniform grids, and the well-balance property significantly improve the numerical accuracy

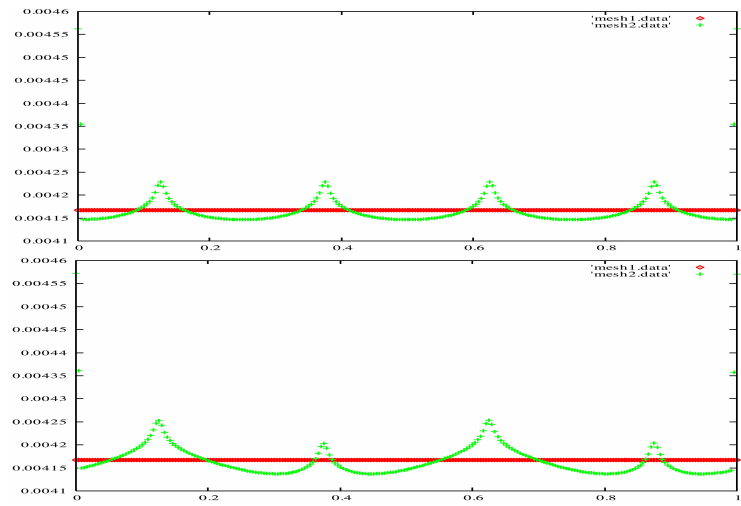

Figure: cells sizes after mesh refinement (green) over uniform mesh

- C. Arvanitis, Mesh redistribution strategies and finite element schemes for hyperbolic conservation laws, J. Sci. Comput. (2008) 


\begin{tabular}{|c|c|c|c|c|c|c|}
\hline cells & \multicolumn{2}{|c|}{$\|e(t)\|_{L^{1}}$} & \multicolumn{2}{c|}{$\|e(t)\|_{L^{2}}$} & \multicolumn{2}{c|}{$\|e(t)\|_{L_{\infty}}$} \\
\hline & & rates & & rates & & rates \\
\hline 30 & $0.195000 \mathrm{E}-02$ & & $0.232276 \mathrm{E}-02$ & & $0.419508 \mathrm{E}-02$ & \\
\hline 60 & $0.569572 \mathrm{E}-01$ & 1.820 & $0.679157 \mathrm{E}-01$ & 1.819 & $0.123060 \mathrm{E}-02$ & 1.814 \\
\hline 120 & $0.218606 \mathrm{E}-01$ & 1.619 & $0.260709 \mathrm{E}-01$ & 1.618 & $0.470566 \mathrm{E}-01$ & 1.619 \\
\hline 240 & $0.960606 \mathrm{E}-00$ & 1.484 & $0.114540 \mathrm{E}-01$ & 1.484 & $0.206341 \mathrm{E}-01$ & 1.485 \\
\hline 480 & $0.450752 \mathrm{E}-00$ & 1.391 & $0.537440 \mathrm{E}-00$ & 1.391 & $0.967720 \mathrm{E}-00$ & 1.392 \\
\hline 960 & $0.221537 \mathrm{E}-00$ & 1.234 & $0.294508 \mathrm{E}-00$ & 1.233 & $0.461213 \mathrm{E}-00$ & 1.234 \\
\hline
\end{tabular}

Table: experimental well-balance error $=0.224508 E-02$

- the well-balance error is predominant for finer grids, and higher rates are observed at longer times for the smoothing effects of mesh refinement - difficulties are undervalued in the simulation of stationary solutions, so that testing the convergence rates may not be really effective...

- counter-examples for well-balanced schemes with centered fluxes on strongly or adaptive nonuniform meshes (uniform block-structured grids) - (theoretical) convergence does not mean accurate pointwise simulation 
the general (finite volume) Upwind Source at Interface scheme reads

$$
\frac{\Delta x_{i}}{\Delta t_{n}}\left(v_{i}^{n+1}-v_{i}^{n}\right)+\left(A_{i+\frac{1}{2}}^{n}-A_{i-\frac{1}{2}}^{n}\right)+B_{i-\frac{1}{2}}^{n,+}+B_{i+\frac{1}{2}}^{n,-}=0
$$

and the numerical source term does not take a conservative form,

$$
B_{i+\frac{1}{2}}^{n, \pm}=B^{ \pm}\left(\Delta x_{i}, \Delta x_{i+1}, v_{i}^{n}, v_{i+1}^{n}, z_{i+1}-z_{i}\right)
$$

the minimal (global) consistency requirement, for $K_{B}>0$ constant,

$$
\left|\frac{B^{-}(h, k, u, u, \lambda)+B^{+}(h, k, u, u, \lambda)}{\lambda}-b(u)\right| \leq K_{B} \lambda
$$

is actually a structural property (not derived from the modified equation) and thus it could fail the truncation error to vanish for nonuniform grids

- B. Perthame, C. Simeoni, Convergence of the upwind interface source method for hyperbolic conservation laws, Hyperbolic problems: theory, numerics, applications, 61-78, Springer, Berlin, 2003 (invited paper)

well-balanced schemes are consistent (Godunov solvers, VFRoe schemes, relaxation and central methods, kinetic schemes) for the supra-convergence

- F. Bouchut, Nonlinear stability of finite volume methods for hyperbolic conservation laws and well-balanced schemes for sources, Frontiers in Mathematics, Birkhäuser (2004) 
influence of the non-uniformity of grids on the convergence's rates $=$ effect of grid irregularity on the accuracy of finite volume algorithms

- alternative technique for rigorous proofs, because entropy methods following the Kružkov's theory do not provide the rates of convergence (and BV-bounds for strong convergence hold uniquely for uniform grids)

- M.G. Crandall, A. Majda, Monotone difference approximations for scalar conservation laws, Math. Comp. (1980)

- R. Sanders, On convergence of monotone finite difference schemes with variable spatial differencing, Math. Comp. (1983)

- thanks to an explicit formulation in terms of numerical derivatives, mesh-dependent extensions of the Lax-Wendroff method (on staggered grids) retrieve conservation form and standard (local) consistency

- M. Dumbser, A. Hidalgo, M. Castro, C. Parés, E.F. Toro, FORCE schemes on unstructured meshes II : Non-conservative hyperbolic systems, Comput. Methods Appl. Mech. Engrg. (2010) 